\title{
Desenvolvimento de uma Ferramenta para Auxílio ao Diagnóstico de Catarata em Telemedicina
}

\author{
R. Husemann; M. Negreiros; H. Tomaggi \\ Departamento de Engenharia Elétrica \\ UFRGS \\ Porto Alegre, Brasil \\ \{ronaldo.husemann, marcelo.negreiros, \\ iop.tomaggi\}@ufrgs.br
}

\begin{abstract}
The use of videoconferencing technology for health systems has the potential to increase the number of patients treated while decreasing cost and allowing distributed local consultation facilities close to the target communities, minimizing the need for transportation and minimizing waiting times. One such initiative is the Teleoftalmo-RS videocolaboration program where ophthalmologic consultations are performed. In this paper, the development of an auxiliary tool to help expedite the diagnosis of cataracts in such a system is presented. This work is also part of the ongoing RNP and Microsoft Challenge: Artificial Intelligence R\&D.
\end{abstract}

\section{KEYWORDS}

Telemedicine, Telediagnosis, Videocolaboration, Cataract

\section{Introdução}

O uso de sistemas de telemedicina e telediagnóstico permite otimizar a operação dos sistemas de saúde. A telessaúde constitui-se numa nova forma de entregar práticas tradicionais, como no caso da teleconsultoria e da teleducação, e como uma maneira alternativa e substitutiva no caso de telediagnóstico e da teleconsulta com potencial de ser o eixo organizador dos sistemas de saúde em nível nacional [1].

O projeto TelessaúdeRS/UFRGS foi estabelecido em 2007 [2]. Como um programa mais recente dessa iniciativa encontra-se o projeto Teleoftalmo: Olhar Gaúcho, que efetua exames oftalmológicos em cerca de 1000 pacientes do Sistema Único de Saúde (SUS) a cada mês através de uma ferramenta própria de vídeo colaboração. O sistema permite a interação em tempo-real entre médicos especialistas e pacientes em centros remotos no Rio Grande do Sul [3,4]. Utilizando essa ferramenta, o médico especialista pode conversar com os pacientes e realizar exames oftalmológicos por equipamentos instalados operados à distância conforme ilustra a figura 1.

In: VI Workshop "O Futuro da Videocolaboração" (WCT-Video 2019), Rio de Janeiro, Brasil. Anais Estendidos do Simpósio Brasileiro de Sistemas Multimídia e Web (WebMedia). Porto Alegre: Sociedade Brasileira de Computação, 2019. (C)2019 SBC - Sociedade Brasileira de Computação. ISSN: 2596-1683

\author{
Aline Lutz Araujo \\ Telessaúde-RS \\ UFRGS \\ Porto Alegre, Brasil \\ tsrs-aline.araujo@ufrgs.br
}

\author{
Valter Roesler \\ Instituto de Informática \\ UFRGS \\ Porto Alegre, Brasil \\ roesler@inf.ufrgs.br
}

Catarata é a principal causadora de perda de visão (cegueira) em nível mundial e é um dos principais fatores que levam à diminuição visual no mundo [5-6]. Não há tratamento médico que possa prevenir a formação ou a progressão da catarata no cristalino. Embora vários fatores de risco para o desenvolvimento de catarata tenham sido identificados, tais como idade, composição genética, luz ultravioleta e diabetes, dados para prevenir cataratas ainda não são conclusivos [7].

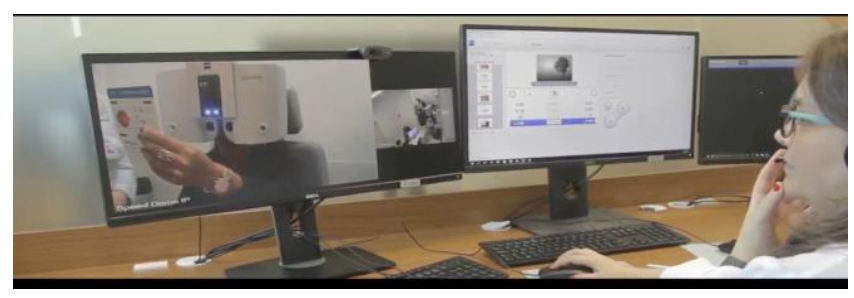

Figura 1: Atendimento do Teleoftalmo-RS

O fator de risco mais importante para catarata é a idade. Cataratas relacionadas à idade constituem a grande maioria de todos os tipos de cataratas e se constituem em um grande problema de saúde pública em nível mundial [8]. Como o número de pessoas afetadas por problemas de visão tem crescido nos últimos anos, necessita-se de um grande aumento de atendimento nos serviços médicos para minimizar os problemas de visão [9]. A crescente demanda de avaliações oftalmológicas traz consigo a necessidade de implementação de soluções que agilizem o tempo de análise e emissão de laudos de exames.

A Academia Americana de Oftalmologia (AAO) posiciona-se favoravelmente ao uso de tecnologias na especialidade, incluindo métodos para rastreamento, refração e outros testes diagnósticos baseados na Internet, desde que obedecidos os mesmos critérios usados pelos exames médicos [10].

Sistemas que utilizam inteligência artificial têm se mostrado capazes de detectar diferentes doenças a partir de imagens e dados médicos [11], tais como melanoma [12] e retinopatia diabética [13]. Nestes exemplos, a performance do sistema é comparável à interpretação diagnóstica feita por médico especialista na respectiva área. Sistemas de graduação automática de catarata provaram ser uma maneira reprodutível e 
objetiva, embora ainda com limitações e necessitando de refinamento [14].

O presente trabalho propõe a utilização de técnicas de inteligência artificial para processar imagens oftalmológicas de exames de retroiluminação e auxiliar no diagnóstico da doença catarata, obtendo uma classificação automática entre paciente normal ou com catarata, agilizando a tomada de decisão do médico, com o objetivo de integrar o sistema presente no TeleoftalmoRS.

Este trabalho será estruturado com descrito a seguir. Na seção 2 será apresentada uma revisão de trabalhos da literatura relevantes para o assunto. A seção 3 traz a metodologia usada no trabalho. Na seção 4 se apresentam os resultados preliminares deste trabalho, que faz parte dos projetos aprovados no edital RNP-Microsoft de Inteligência Artificial para a área de Videocolaboração.

\section{Revisão da literatura}

A seção atual traz uma revisão da literatura com trabalhos relacionados à detecção e graduação automática de imagens de catarata. A catarata somente é clinicamente relevante se a capacidade visual do paciente for afetada significativamente, uma vez que opacidade no cristalino pode existir sem qualquer sintoma [7]. Existem três tipos principais de catarata relacionada à idade: nuclear, cortical e subcapsular posterior, de acordo com a localização da opacidade no cristalino. Em muitos pacientes mais de um tipo de catarata estão presentes ao mesmo tempo [7,9]. A figura 2 apresenta os tipos mais comuns de cataratas senis, incluindo a catarata em estágio avançado ou madura [15].

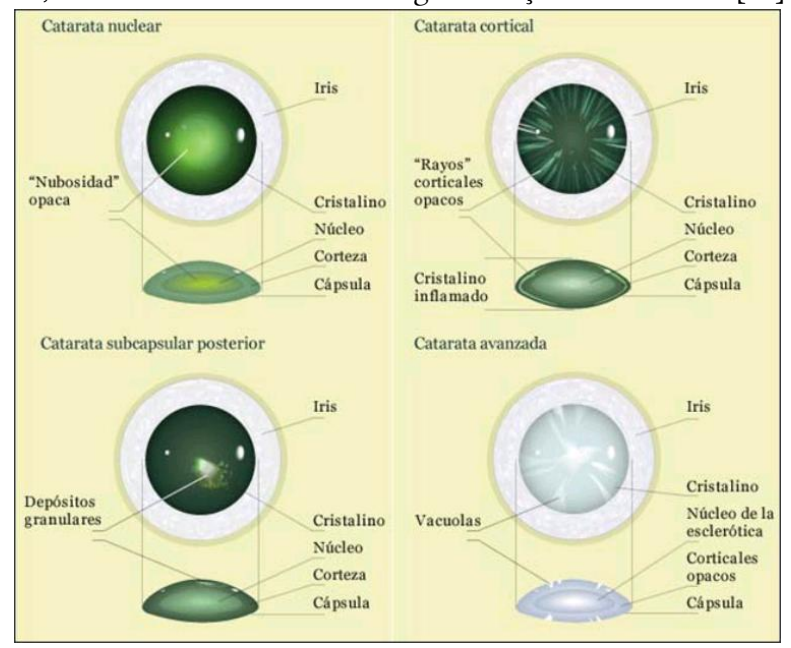

Figura 2: Morfologia de catarata senil [15]

Imagens obtidas pelo exame de lâmpada de fenda são usadas para avaliação de opacidades nucleares. No entanto essas imagens não são adequadas para avaliar opacidades corticais e subcapsulares, mais nítidas em imagens de retroiluminação que se utilizam da característica do fundo do olho humano de refletir a luz vermelha com maior intensidade [16]. Nos trabalhos presentes na literatura são usados polarizadores cruzados para a eliminação dos reflexos na córnea causados pela iluminação intensa durante o exame de retroiluminação, necessitando portanto de equipamento especial [16]. O aspecto das imagens dos exames de lâmpada de fenda e de retroiluminação é apresentado na figura 3. Note que a área mostrada nas imagens da segunda e terceira linhas é a pupila, normalmente dilatada durante o exame para permitir a avaliação da maior área possível do cristalino.

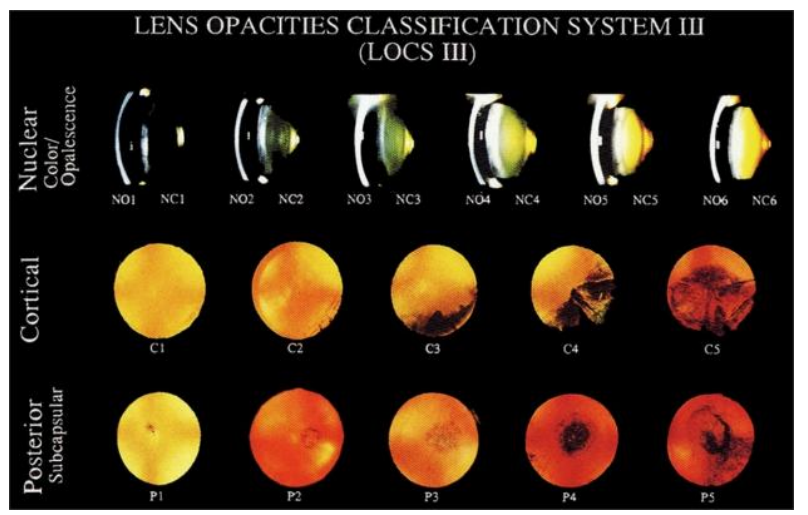

Figura 3: Sistema LOCS III de graduação de opacidades [17]

Esquemas para a classificação objetiva de catarata usam padrões fotográficos para subdividir os principais tipos em diferentes graus, conforme exemplificado na figura 3. Esses graus podem ser baseados na densidade e na cor (no caso do núcleo) ou de acordo com a área anatômica da catarata (no caso de áreas cortical ou subcapsular posterior). Dentre os esquemas objetivos de classificação para cataratas podem ser citados: Lens Opacities Classification System II and III (LOCS II and LOCS III), Oxford Cataract Classification System, Beaver Dam Eye Study e Age Related Eye Diseases Study (AREDS) [8,14,17,18]. A capacidade visual do paciente é afetada de modo diferente de acordo com o tipo e grau de catarata. Segundo [19], usando o sistema LOCS III, cataratas nucleares de graus 4 e 5 afetaram a capacidade visual para opalescência e cor, respectivamente. Cataratas corticais afetaram a capacidade visual no grau 3, e cataratas posterior subcapsulares afetaram a capacidade visual em todos os graus.

A graduação manual por especialistas é um processo demorado e caro, além de possuir baixa reprodutibilidade e ser sujeito à inconsistências por fadiga [15,20]. A graduação automática tem a possibilidade de aumentar a reprodutibilidade, objetividade e velocidade do exame, bem como diminuir seu custo.

Um sistema para medição objetiva de opacidade cortical e subcapsular em imagens de exames de retroiluminação com o auxilio de computador foi proposto por Wolfe em 1990 [21], no entanto necessitava de intervenção de um operador para indicação de áreas opacas e claras na imagem. Harris [16] propôs um método para correção de variação de iluminação em exames de retroiluminação. Alguns sistemas de detecção automática de cataratas tem sido propostos [20,22,23] mas com problemas com cataratas em estágio inicial ou muito severas. Em [24] foi proposto um sistema de graduação baseado no método de 
Desenvolvimento de uma Ferramenta para Auxílio ao Diagnóstico de Catarata em Telemedicina

aprendizado supervisionado SVR. Mais recentemente, uma abordagem usando deep learning para graduação de catarata nuclear baseada em imagens de exames de lâmpada de fenda foi apresentada em [25]. Em [26] uma abordagem baseada em deep learning e imagens de fundo de olho mostrou bons resultados na detecção de estágios iniciais de catarata. Um panorama geral da pesquisa na área é apresentado em [14, 15].

\section{Metodologia}

Nessa seção é descrita a metodologia usada no desenvolvimento do trabalho, desde a preparação do banco de dados de imagens até as demais etapas de processamento.

O banco de dados é composto por imagens de exames oftalmológicos de retroiluminação. As imagens são obtidas com pupila dilatada, obtidas através de retinógrafo Zeiss Visucam 224 , com resolução de pelo menos $2448 \times 2448$ pixels, ângulo $30^{\circ}$, flash 6 e foco manual na íris. $O$ aspecto das imagens é apresentado na figura 4 (notar similaridade com figura 2). Existe reflexo da córnea (notar flash ou círculo branco no centro das pupilas) nas imagens porque o retinógrafo não é equipado com polarizadores.



(a)

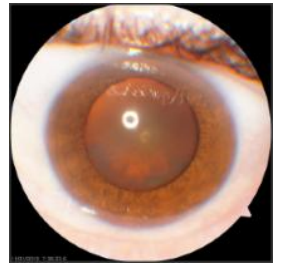

(b)



(d)

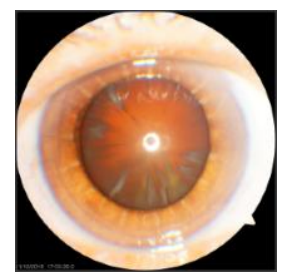

(c)

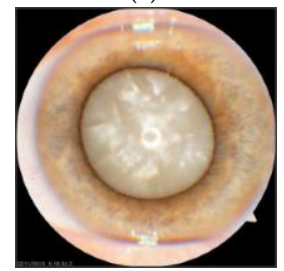

(e)
Figura 4: Imagens típicas no banco de dados: (a) sem catarata, catarata (b) nuclear, (c) cortical, (d) posterior subcapsular e (e) avançada ou madura

O processamento de dados está dividido em duas etapas: identificação de parte de interesse da imagem e classificação da doença.

A primeira etapa tem por finalidade segmentar a área de interesse, a pupila do olho, das demais regiões da imagem como íris, detalhes da córnea, pálpebras, cílios e pele. A implementação da primeira etapa inclui algoritmos de segmentação, detecção de borda e formato. São avaliados diferentes canais de cores das imagens para segmentar a região da pupila. Como as imagens possuem diferentes intensidades de iluminação, contraste e zoom, bem como cores diferentes na íris e na pupila, não existe
Anais Estendidos do WebMedia'2019, Rio de Janeiro, Brasil

um canal de cor ideal. Deste modo são analisados quatro canais distintos (saturação, luminância, crominância vermelha e cor vermelha), os resultados são avaliados e é escolhida uma das soluções. Dentre as técnicas usadas podem ser citadas a detecção de bordas (Canny) e a transformada de Hough. Dentre os critérios para escolha do melhor resultado estão a proximidade do centro da imagem, o raio do círculo e o percentual do círculo contido na borda detectada. A saída da primeira etapa é o ponto central do circulo que aproxima o contorno da pupila em coordenadas $(\mathrm{x}, \mathrm{y})$ bem como o raio deste círculo. Uma ilustração do método se apresenta na Figura 5.

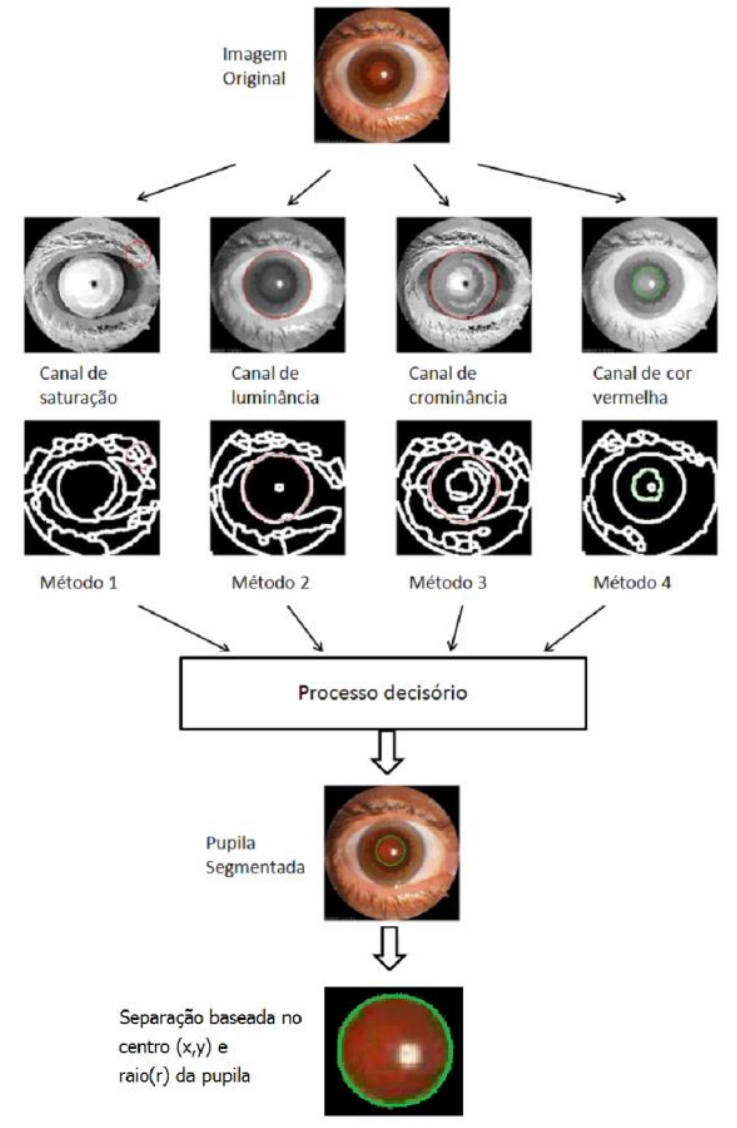

Figura 5: Algoritmo de segmentação de pupila

A segunda etapa tem por finalidade mensurar a opacidade no cristalino (lente do olho) através da análise da imagem segmentada da pupila e indicar a presença ou não de catarata. $\mathrm{O}$ objetivo do projeto é usar uma rede de aprendizagem profunda baseada em redes neurais do tipo convolutivas (CNNs) de modo similar a outros trabalhos na área $[14,25,26]$.

\section{Resultados preliminares}

Os resultados alcançados até o momento referem-se à validação do algoritmo de segmentação de pupila rodando sobre uma versão maior do banco de dados de imagens, bem como do 
desenvolvimento de uma versão do banco de dados para uso com treinamento de redes neurais.

Como o banco de dados não foi desenvolvido primariamente para o trabalho acadêmico existe a necessidade de filtrar as imagens mais adequadas e representativas da doença catarata. Um número semelhante de imagens com e sem a doença é necessário para a etapa de treinamento da rede neural. Desse modo o banco de dados conta atualmente com 1161 imagens de exames de retroiluminação com qualidade julgada adequada para diagnóstico pela equipe de oftalmologistas (grupo 1). Outras 1544 imagens do banco apresentaram problemas na aquisição de dados e foram consideradas inadequadas para o exame (grupo 2). Um grupo adicional com mais de 2000 imagens de exames recentes aguarda análise de qualidade (grupo 3). Os problemas de aquisição incluem imagens fora de foco, com obstrução parcial ou total (pálpebra), outras patologias da córnea que podem causar opacidade, pouca dilatação da pupila e olhos com lentes artificiais intraoculares (após cirurgia de catarata).

Os resultados do algoritmo de detecção de pupila, para o banco de dados atual, separado pelos grupos citados no parágrafo anterior, encontram-se na Tabela 1.

Tabela 1: Resultados de segmentação de pupila

\begin{tabular}{|c|c|c|c|c|}
\hline Grupo & Total & Acertos & Erros & Percentual de Acertos \\
\hline 1 & 1161 & 1071 & 90 & $92,2 \%$ \\
\hline 2 & 1544 & 1380 & 164 & $89,4 \%$ \\
\hline 3 & 2156 & 1923 & 233 & $89,2 \%$ \\
\hline
\end{tabular}

\section{Considerações Finais}

O desenvolvimento de uma versão do banco de dados adequada ao treinamento de redes neurais está em desenvolvimento com a possibilidade de ser continuamente expandido ao longo do desenvolvimento do projeto e no futuro.

No contexto de redes neurais e deep learning o desenvolvimento de um banco de dados adequado e com milhares de imagens é essencial. Os resultados de segmentação de pupila mostraram-se adequados aos perfis das imagens, com menor taxa de acerto no subconjunto de imagens com problemas de aquisição.

Estima-se para os próximos anos por um aumento significativo do uso de tecnologia de deep learning em aplicações médicas, principalmente no campo de diagnóstico e telediagnóstico, se prevendo os seguintes benefícios potenciais: aumento do acesso aos serviços de saúde, maior envolvimento do usuário no seu processo de saúde e de tomada de decisão relativa à saúde, aumento da eficiência na prestação de serviços e diminuição dos custos em saúde .

\section{AGRADECIMENTOS}

Este trabalho está sendo patrocinado pela RNP (Rede Nacional de Ensino e Pesquisa) e pela empresa Microsoft.

\section{REFERÊNCIAS}

[1] Harzheim, Erno et al. Telessaúde como eixo organizacional dos sistemas universais de saúde do século XXI. Revista Brasileira de Medicina de Família e Comunidade, [S.1.], v. 14, n. 41, p. 1881, fev. 2019. ISSN 2179-7994. doi:https://doi.org/10.5712/rbmfc14(41)1881.

[2] Harzheim, E. et al., Telehealth in Rio Grande do Sul, Brazil: Bridging the Gaps. Telemedicine Journal and e-Health, v. 22, p. 1-7, 2016. doi: $10.1089 / \mathrm{tmj} .2015 .0210$

[3] Projeto TelessaúdeRS - Teleoftalmo. Online: https://www.ufrgs.br/telessauders/telediagnostico/teleoftalmo/

[4] Roesler V., Harzheim E. Tecnologias emergentes em telemedicina - ênfase em videocolaboração In Anais of XXIII Webmedia WCT-Video. 19de outubro, 2017, Gramado, Rio Grande do Sul. 368p. E-book e Impresso. ISBN: 978-857669-381-9.

[5] Pascolini D, Mariotti SP, Global estimates of visual impairment: 2010,British $\begin{array}{llll}\text { Journal of } & \text { Ophthalmology 2012; 96: }\end{array}$ http://dx.doi.org/10.1136/bjophthalmol-2011-300539

[6] Resnikoff, S., Pascolini, D., Etya'ale, D., Kocur, I., Pararajasegaram, R., Pokharel, G. P., \& Mariotti, S. P. (2004). Global data on visual impairment in the year 2002. Bulletin of the World Health Organization, 82(11), 844-51.

[7] Asbell PA, Dualan I, Mindel J, et al. Age-related cataract. Lancet, 2005, vol. 365, p.599-609. https://www.thelancet.com/journals/lancet/article/PIIS01406736(05)17911-2/fulltext

[8] Malhotra, 2008, Cataract,Raman Elsevier Chapter 1: The ageing lens and classification of cataracts

[9] Flaxman SR, et al. Vision Loss Expert Group of the Global Burden of Disease Study. Global causes of blindness and distance vision impairment 1990-2020: a systematic review and meta analysis. Lancet Glob Health. 2017;5(12):e1221e1234.

[10] American Academy of Ophthalmology. Information statement: Use of innovative technologies in diagnosing eye diseases and conditions. September 2014. https://www.aao.org/clinical-statement/innovative-technologies-indiagnosing-eye-diseases

[11] Rajkomar A, Oren E, Chen K, et al. Scalable and accurate deep learning with electronic health records. Npj Digital Medicine. 2018; 18. doi:10.1038/s41746018-0029-1

[12] Esteva A, Kuprel B, Novoa RA, et al. Dermatologist-level classification of skin cancer with deep neural networks. Nature. 2017;542:115-118.

[13] Gargeya R, Leng T. Automated identification of diabetic retinopathy using deep learning. Ophthalmology. 2017;124: 962-969.

[14] Helena E. Gali, Ruti Sella, and Natalie A. Afshari, Cataract grading systems: a review of past and present, Curr Opin Ophthalmol 2019, 30:13-18 DOI:10.1097/ICU.0000000000000542

[15] H. I. M. Lopez, J. C. S. Garcia, J. A. D. Méndez, Cataract Detection Techniques: A Review. IEEE LATIN AMERICA TRANSACTIONS, VOL. 14, NO. 7, JULY.2016, pp. 3074-3079.

[16] M. L. Harris et al.. Analysis of Retro-Illumination Photographs for Use in Longitudinal Studies of Cataract. Eye (1993) 7,572-577.

[17] Chylack LT, Wolfe JK, Singer DM, et al. The lens opacities classification system III. Arch Ophthalmol 1993; 111:831-836.

[18] Age-Related Eye Disease Study Research Group. The age-related eye disease study (AREDS) system for classifying cataracts from photographs: AREDS report no. 4. Am J Ophthalmol. 2001;131(2):167-175.

[19] Chew M, Chiang PPC, Zheng Y, et al. The impact of cataract, cataract types, and cataract grades on vision-specific functioning using rasch analysis. Am J Ophthalmol 2012; 154:29.e2-38.e2

[20] Y. C. Chow et al. Automatic Detection of Cortical and PSC Cataracts Using Texture and Intensity Analysis on Retro-illumination Lens Images. 33rd Annual International Conference of the IEEE EMBS Boston, Massachusetts USA, August 30 - September 3, 2011, pp 5044-5047.

[21] Wolfe JK, Chylack LT Jr: Objective measurement of cortical and subcapsular opacification in retroillumination photographs. Ophthalmic Res 1990;22(suppl 1):62-67.

[22] H. Li et al., "Image Based Diagnosis of Cortical Cataract," Proceedings of Annual International Conference of the IEEE Engineering in Medicine and Biology Society, 2008.

[23] H. Li et al., Automatic Detection of Posterior Subcapsular Cataract Opacity for Cataract Screening, 32nd Annual International Conference of the IEEE EMBS Buenos Aires, Argentina, August 31 - September 4, 2010, pp. 5359-5362.

[24] Gao X. et al., (2013) Automatic Grading of Cortical and PSC Cataracts Using Retroillumination Lens Images. In: Lee K.M., Matsushita Y., Rehg J.M., Hu Z. (eds) Computer Vision - ACCV 2012. ACCV 2012. Lecture Notes in Computer Science, vol 7725. Springer, Berlin, Heidelberg. doi:https://doi.org/10.1007/978 3-642-37444-9_20.

[25] Gao SL X, TYW. Automatic feature learning to grade nuclear cataracts based on deep learning. IEEE Trans Biomed Eng 2015; 62:2693-2701.

[26] Li J. et al. Automatic Cataract Diagnosis by Image-Based Interpretability. 2018 IEEE International Conference on Systems, Man, and Cybernetics. p. 39643969. DOI 10.1109/SMC.2018.00672. 